\title{
On-chip wireless silicon photonics: from reconfigurable interconnects to lab-on-chip devices
}

\author{
Carlos García-Meca*, Sergio Lechago*, Antoine Brimont, Amadeu Griol, Sara Mas, Luis Sánchez, \\ Laurent Bellieres, Nuria S Losilla and Javier Martí
}

\begin{abstract}
Photonic integrated circuits are developing as key enabling components for high-performance computing and advanced networkon-chip, as well as other emerging technologies such as lab-on-chip sensors, with relevant applications in areas from medicine and biotechnology to aerospace. These demanding applications will require novel features, such as dynamically reconfigurable light pathways, obtained by properly harnessing on-chip optical radiation. In this paper, we introduce a broadband, high directivity $(>150)$, low loss and reconfigurable silicon photonics nanoantenna that fully enables on-chip radiation control. We propose the use of these nanoantennas as versatile building blocks to develop wireless (unguided) silicon photonic devices, which considerably enhance the range of achievable integrated photonic functionalities. As examples of applications, we demonstrate $160 \mathrm{Gbit}^{-1}$ data transmission over mm-scale wireless interconnects, a compact low-crosstalk 12-port crossing and electrically reconfigurable pathways via optical beam steering. Moreover, the realization of a flow micro-cytometer for particle characterization demonstrates the smart system integration potential of our approach as lab-on-chip devices.
\end{abstract}

Light: Science \& Applications (2017) 6, e17053; doi:10.1038/lsa.2017.53; published online 22 September 2017

Keywords: integrated optics; lab-on-a-chip devices; nanoantenna; sensing; silicon photonics

\section{INTRODUCTION}

The advent of photonic integrated circuits (PICs) promises to revolutionize high-performance computing (HPCs) and high-speed on-chip interconnects, because they are able to overcome the limits of conventional electronic technology in terms of speed, bandwidth and energy consumption ${ }^{1}$. In addition, as the possibilities offered by integrated photonics develop, a number of additional utilities, such as biosensing $^{2}$, optical beamforming ${ }^{3}$ or metasurfaces for different applications ${ }^{4}$ are emerging. However, current PIC devices typically rely on fixed layout-guided interconnects that yield stringent design rules and limit network-on-chip reconfigurability, usually leading to complex topologies and large footprints ${ }^{5,6}$. Moreover, the strong confinement of the electromagnetic fields to the guiding structures prevents their interaction with the outer medium at far-field distances, a feature required in a variety of sensors and devices ${ }^{7-9}$, as well as in applications related to microparticle control and acceleration ${ }^{10}$, among others. Just as radio frequency and microwave antenna technologies have enabled countless unprecedented applications (cellular and satellite communications, radar and biomedical imaging, among others), the ability to radiate electromagnetic fields within a chip in a controlled manner would allow us to overcome the aforementioned limitations and open doors to a wide range of new functionalities. As an illustration, envisioned scenarios where on-chip wireless systems could deliver great potential are sketched in Figure 1. Although a wide variety of optical antenna implementations can be found in the literature, they suffer from different shortages that hamper their use as building blocks for the anticipated model of a wireless optical system. Plasmonic nanoantennas, on the one hand, have emerged as a key component in many nanophotonic applications ${ }^{14-19}$ due to their capacity to concentrate optical energy at very high frequencies ${ }^{20}$ and to provide a more efficient alternative to plasmonic wires ${ }^{21-23}$. Unfortunately, they exhibit poor directivity values and high-absorption losses at optical frequencies. In addition, the excitation of these nanoantennas usually requires complex feeding elements such as nanodipoles, off-plane laser sources or quantum $\operatorname{dots}^{14,23,24}$, which in practice represents an additional technological challenge. On the other hand, we can also find studies based on the use of dielectric antennas, employing either arrays of grating couplers ${ }^{25-27}$ or scatterers ${ }^{28}$. However, both configurations are only suitable for off-chip radiation. Furthermore, grating coupler arrays lead to large footprints, whereas scatterers show poor directivity values. In this article, we build on a new concept of silicon optical antennas compatible with complementary metal-oxide-semiconductor (CMOS) technology to show the feasibility of the devised model of an on-chip wireless system. We provide proof of the power of our approach by experimentally demonstrating a diverse range of applications. 
a

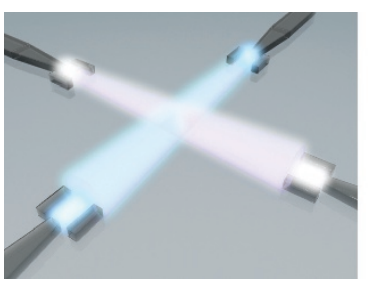

b

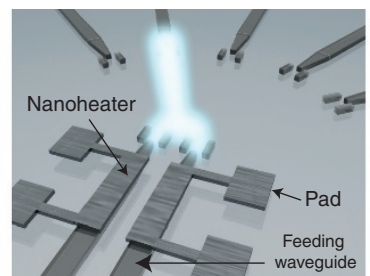

C

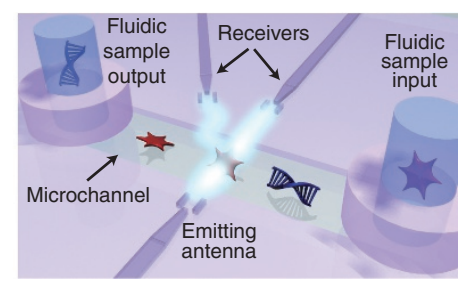

Figure 1 Envisioned scenarios for on-chip wireless optical systems. (a) Flexible networks: the use of highly directive antennas could allow for a variety of interferenceless wireless crossing configurations, favoring more flexible intra- and inter-core communications ${ }^{11,12}$. (b) Reconfigurable pathways and beam shaping: the radiation properties of antenna arrays (for example, radiation direction) depend on each antenna's phase, which can be electrically controlled by modifying the corresponding feeding waveguide temperature (benefiting from silicon's high thermo-optic effect) through the voltage applied over a nanoheater. This could be used for complex on-chip field pattern generation or traffic routing,13. (c) Lab-on-a-chip: the proposed antennas can replace bulky light emitters and receptors, providing an ideal platform for the miniaturization and smart integration of sensors and voluminous lab equipment ${ }^{7,8}$. As an example, we show a fully integrated flow cytometer consisting of a microfluidic channel through which a sample containing several analytes of interest (platelets, DNA segments) can flow and be simultaneously illuminated by an antenna. The scattered light collected by other antennas provides information on each analyte.
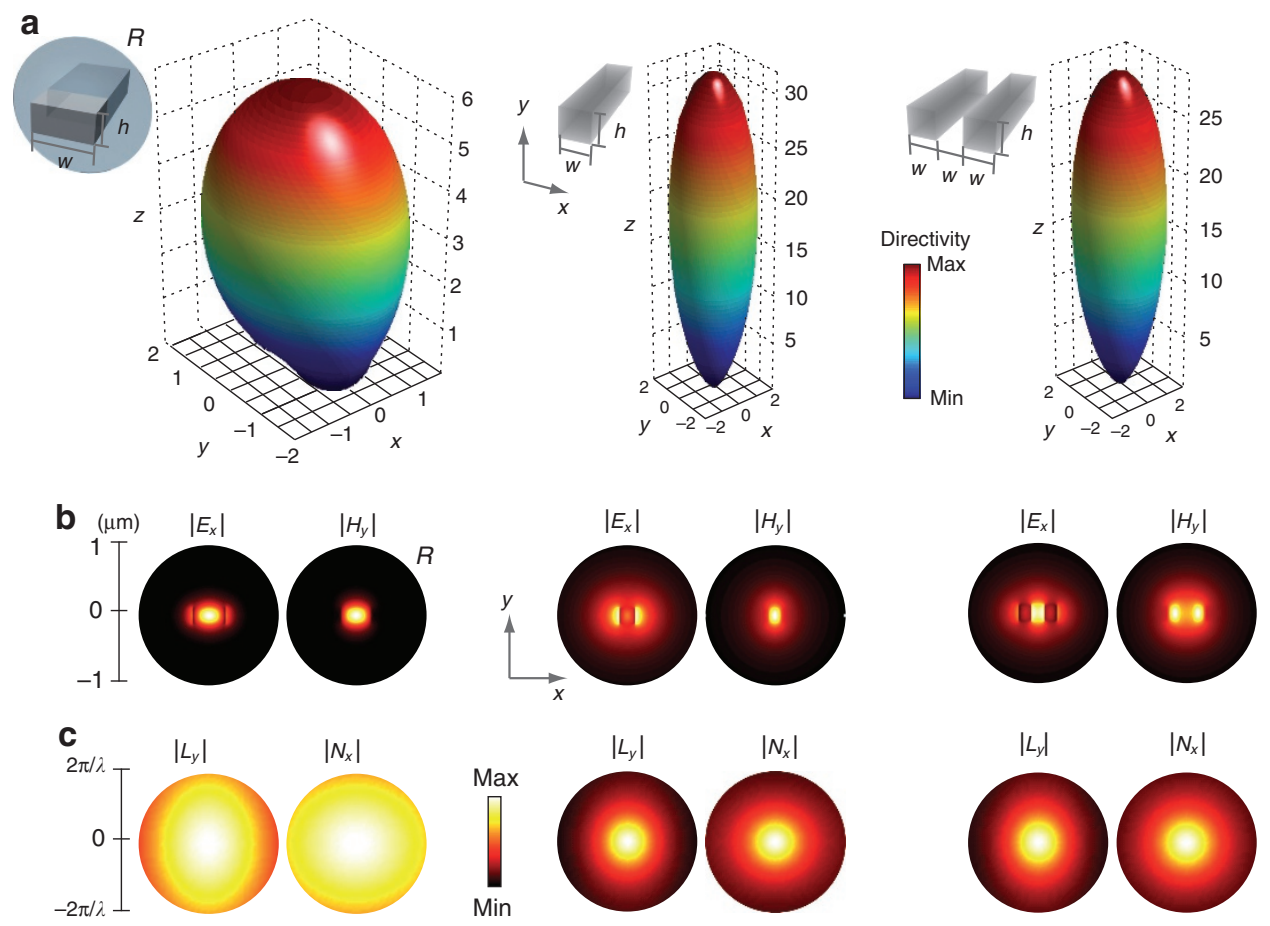

Figure 2 Properties of dielectric waveguides as radiating elements. (a) Directivity diagrams (linear units) of different waveguides with height $h$ and width $w$. (b) Relevant components of the electric and magnetic fields of the modes supported by these waveguides in $R$ (outside this region, the fields are negligible). (c) Corresponding radiation vectors (Supplementary Information). First column: standard strip ( $h=220 \mathrm{~nm}, w=450 \mathrm{~nm})$. Second column: square strip $(h=220 \mathrm{~nm}, w=220 \mathrm{~nm})$. Third column: designed slot $(h=220 \mathrm{~nm}, w=180 \mathrm{~nm})$.

\section{MATERIALS AND METHODS}

\section{Antenna theory and design}

The first step to achieve the desired architecture is to develop a type of antenna exhibiting low loss and low reflection, and whose directivity can be tuned to have a wide range of values able to accommodate different applications, while maintaining a reduced transverse size. In this work, we focus on applications such as mm-scale wireless optical links and on-chip contactless sensing that require very high directivity values $(>50$ ) for good performance. Recent studies show that nanoantennas based on dielectric nanoparticles exhibit lower losses and higher directivity than metallic structures ${ }^{29,30}$, although the directivity is still too low for the above-mentioned applications. Moreover, these structures present important fabrication challenges.
Here, we studied the possibilities of open-ended silicon-on-insulator (SOI) waveguides ${ }^{31}$ as potential radiating elements that could solve these shortcomings. We modeled this type of structure using Huygens' principle $^{32}$, that is, the radiated fields outside a closed surface $S$ surrounding all electromagnetic sources can be obtained from the fields' tangential components on $S$ (Supplementary Information), a technique usually employed to calculate the radiation pattern of microwave metallic aperture antennas. As the fields at the antenna output plane (normal to the propagation direction and containing the waveguide end) are concentrated in a small spatial region $R$, we can apply Huygens' principle by taking $S$ to be this plane, knowing that only the region $R$ will be relevant in the calculations (Figure $2 \mathrm{a}$ and $2 b)$. Moreover, if reflections at the waveguide open end are negligible 
(which is the case in the studied structures), the fields on $S$ are essentially equal to those of the infinite waveguide mode. Hence, this simple approach directly links the waveguide radiation pattern with its two-dimensional (2D) mode profile, which can be easily calculated using a 2D eigensolver. Specifically, the direction-dependent radiation intensity can be obtained straightforwardly from the so-called radiation vectors, which are the Fourier transform of the mode tangential electric and magnetic fields (Supplementary Information). Therefore, less confined modes lead to higher directivities, since, as the spatial extension of the mode increases, the angular extension of the radiation vectors decreases (a property of the Fourier transform); that is, energy is radiated in a smaller set of directions. Remarkably, the mode extension of the studied guiding structures can be readily controlled by varying their transverse dimensions, which gives us an intuitive and simple method for tailoring the antenna directivity. In addition, it can be shown that, from moderately high directivity values, these waveguide-based structures support weakly confined modes that favor impedance matching, resulting in very low reflections. Finally, the use of dielectric materials guarantees the desired low losses. Using these ideas, we designed several structures based on standard SOI photonic waveguides ${ }^{31}$, namely, strip and slot configurations, ensuring a simple fabrication process and avoiding complex feeding structures. The directivity patterns, mode profiles and radiation vectors of several of the studied configurations are depicted in Figure 2. It is clear that a strip waveguide with standard dimensions shows a strong confinement. Consequently, the radiation vector values within the circle of propagating wavenumbers are quite uniform, resulting in a low directivity. However, if the strip width is reduced, the mode profile spreads over a wider region, reducing the angular spectrum extension and enhancing the structure's directivity. For instance, a square strip shows a less confined mode with a directivity of $\sim 30$. Regarding the slot waveguide, we verified that wide-gap versions of this structure propagate less confined modes than standard strip/slot configurations. This analysis allowed us to design a slot waveguide displaying similar directivity values to that of the square strip (Figure 2a).

In practice, we can obtain a reflectionless transition from a standard 450-nm-wide strip waveguide to a custom-width radiating end (exhibiting directivities that can be higher than 100) through an inverted taper. As the elements required for coupling light to the designed slot waveguide usually add larger power penalties to the system, we focused on the strip configuration. For the sake of fabrication simplicity and to avoid long taper structures, we fixed a minimum taper tip width of $150 \mathrm{~nm}$, which allowed us to attain directivities higher than 50. Moreover, inspired by Yagi-Uda antennas ${ }^{16,33}$, we studied the effect of using different planar structures as directors to further improve the directivity of the designed invertedtaper antenna while maintaining a reduced size. As the electromagnetic response of such structures cannot be addressed analytically, its dimensions were determined numerically. Nonetheless, Huygens' method still applies for directivity calculations, although 3D simulations are required to obtain the fields at $R$. Note that in our experiments, the fabricated antennas are covered with a $\mathrm{SiO}_{2}$ layer to improve their performance. The finite size of the resulting $\mathrm{SiO}_{2}$ embedding matrix was also considered in the directivity calculations (Supplementary Information). After an optimization process (Supplementary Information), we obtained a director configuration consisting of two symmetric silicon strips. In addition, we analyzed the effect of using several directors (up to three), whose separation was optimized as well (Figure 3b). The resulting antenna (inverted taper +directors, Figure 3a) can be engineered to display directivity values between 5 and 150, providing an extremely high design flexibility. The directivity improvement associated with the third director is moderate a

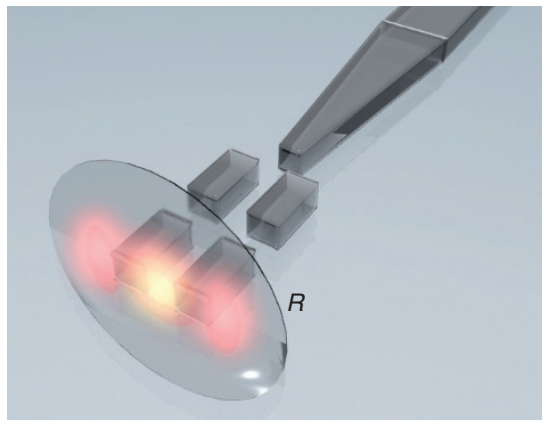

C

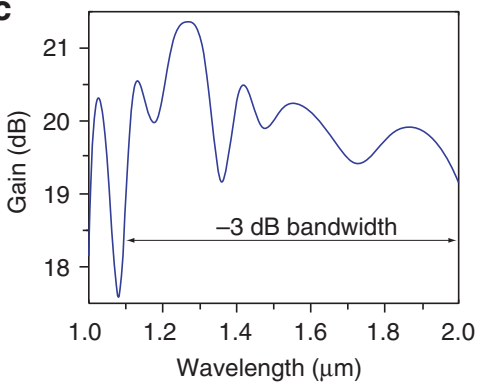

b
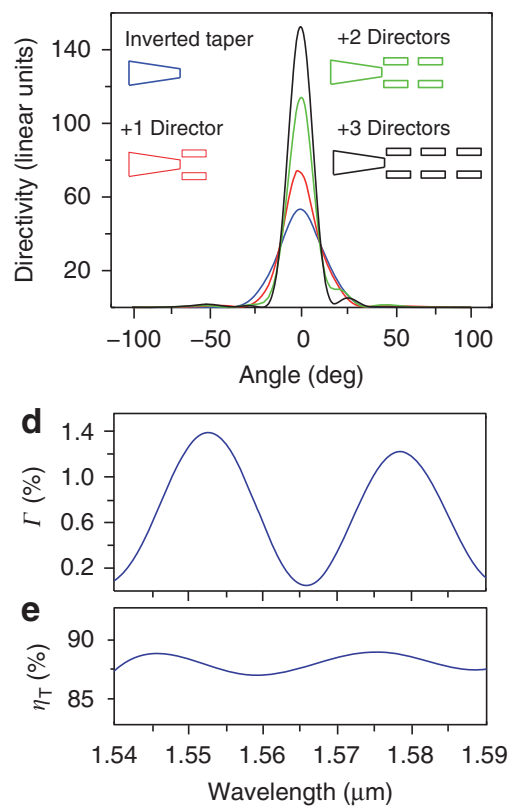

Figure 3 Designed antenna and main parameters. (a) Illustration of the inverted-taper antenna with two directors and the fields in the region $R$ employed in Huygens' method. (b) Directivity of the designed antenna as a function of the number of directors. (c) Theoretical antenna gain and - 3 dB bandwidth. (d) Simulated reflectance $\Gamma$ within the window of the experimental measurements. (e) Total efficiency $\eta_{\mathrm{T}}=\eta_{\mathrm{R}}\left(1-|\Gamma|^{2}\right)$, where $\eta_{\mathrm{R}}$ is the antenna radiation efficiency. Calculations were performed with CST Microwave Studio. 
( 33\%) compared to the improvement achieved by the second director $(\sim 54 \%)$. For simplicity, we chose a two-director configuration (directivity of 114), which already satisfied the requirements for all the considered applications. Note that recent dielectric antennas reach directivity values up to 12 (Ref. 29), whereas typical plasmonic nanoantennas exhibit lower values ${ }^{21-23}$. The designed antennas have another important feature: a large bandwidth. Specifically, the calculated gain of the designed antenna exhibits a $-3 \mathrm{~dB}$ bandwidth higher than $900 \mathrm{~nm}$ (Figure 3c). The reason is that these structures do not rely on resonant phenomena. In contrast, they display almost perfect impedance matching in a wide spectral region, thus yielding low reflections (Figure 3d) as well as a total efficiency close to $90 \%$ (Figure 3e). Therefore, unlike in typical plasmonic works, auxiliary resonant matching elements are not required, avoiding the bandwidth limitation they introduce. Let us now see how the proposed low loss, broadband and highly directive antenna configuration can provide efficient solutions in many different situations.

\section{Fabrication of the optical devices}

The antenna-based links were fabricated on standard SOI samples from SOITEC (Bernin, France) wafers with a top silicon layer thickness of $220 \mathrm{~nm}$ (resistivity $\rho \sim 1-10 \Omega \mathrm{cm}^{-1}$, with a lightly $p$-type background doping of $\sim 10^{15} \mathrm{~cm}^{-3}$ ) and a buried oxide layer thickness of $2 \mu \mathrm{m}$. The fabrication is based on an electron-beam direct-writing process performed on a coated $100 \mathrm{~nm}$ hydrogen silsesquioxane (HSQ) resist film. The mentioned electron-beam exposure, performed with a Raith150 tool, was optimized to reach the required dimensions employing an acceleration voltage of $30 \mathrm{KeV}$ and an aperture size of $30 \mu \mathrm{m}$. After developing the HSQ resist using tetramethylammonium hydroxide, the resist patterns were transferred onto the SOI samples employing an optimized inductively coupled plasma-reactive ion etching process with fluoride gases. Finally, a $2 \mu \mathrm{m}$-thick silicon dioxide upper cladding was deposited on the SOI sample by using a plasma-enhanced chemical vapor deposition system from Applied Materials (except in the beam-steering experiment, in which a $1 \mu \mathrm{m}$-thick layer was used to improve the efficiency of the nanoheaters).

\section{Fabrication of the nanoheaters}

The nanoheaters were fabricated using a direct writing electron-beam process to transfer the patterns onto a 300-nm-thick Poly-methyl methacrylate resist layer. The thin titanium layer evaporation was performed prior to a lift-off process using $N$-methyl-2-pyrrolidone. The high thermo-optic coefficient of silicon enables large refractive index $(n)$ changes produced by local temperature $(T)$ variations $\left(\partial n / \partial T=1.86 \times 10^{-4} \mathrm{~K}^{-1}\right)$. As an example, a temperature variation of only $10{ }^{\circ} \mathrm{C}$ (readily available in our configuration) can lead to a local refractive index variation of $\Delta n=1.86 \times 10^{-3}$, implying that a full $\pi$-phase shift can be achieved over a length of $L_{\pi}=417 \mu \mathrm{m}$, assuming a uniform distribution of the heat across the waveguide. In our configuration, we employed $1.5-\mathrm{mm}$-long heaters to ensure full control over the phase. Interestingly, because the thermo-optic effect only affects the real part of the refractive index, current-dependent absorption mechanisms, which could affect the output intensity of each individual antenna (and hence create distortion of the overall radiation pattern), do not take place. This is in contrast to the widely used free-carrier plasma dispersion effect, where both the real and imaginary parts of the refractive are altered.

\section{Fabrication of the microfluidic system}

For the fabrication of the fluidic channel, a Cr layer of $35 \mathrm{~nm}$ was first deposited on the SOI sample by using electron-beam metal evaporation. A direct writing electron-beam exposure of the channel was then performed on a layer of $100 \mathrm{~nm}$ of PMMA 950K positive resist. After the resist development, $\mathrm{Cr}$ was removed from the channel area using a wet $\mathrm{Cr}$ etchant process based on $\mathrm{CR}-7\left(\mathrm{HClO}_{4}+\mathrm{C}\left(\mathrm{NH}_{4}\right)_{2}\right.$ $\left.\left(\mathrm{NO}_{3}\right)_{6}+\mathrm{H}_{2} \mathrm{O}\right)$. Afterwards, an ICP-RIE process was carried out to open the channel through the $\mathrm{SiO}_{2}$. Finally, the sample was cleaned to remove the organic residues using a mixture of $\mathrm{H}_{2} \mathrm{SO}_{4}$ and $\mathrm{H}_{2} \mathrm{O}_{2}(3: 1)$ for $20 \mathrm{~min}$ and then washed by deionized water (DIW). This cleaning procedure was also used to regenerate the device after sensing experiments. To prepare the employed PDMS thin substrates (Sylgard 184 Silicone Elastomer Down, Corning), the PDMS mixture (10:1) was spin-coated on glass cover slides and cured at $60^{\circ} \mathrm{C}$ for $1 \mathrm{~h}$ to obtain a thin $(150 \mu \mathrm{m})$ PDMS layer on the glass slide. After the curing process, the PDMS was peeled off from the glass slide and washed in absolute ethanol to clean the surface. Finally, the PDMS substrate was positioned and aligned on top of the fluidic channel. The polystyrene microparticles used in the experiments were obtained from SigmaAldrich and have a size of 1 and $2 \mu \mathrm{m}$ with a s.d. $<0.05 \mu \mathrm{m}$.

\section{RESULTS AND DISCUSSION}

As an initial demonstration, we experimentally achieved the first wireless transmission through on-chip links. Moreover, we demonstrated high-speed data transmission over mm-scale distances. For this purpose, several links consisting of two identical antennas (see Figure $4 \mathrm{~d}$ for final antenna dimensions) with different gap distances $d(0.1,0.2,0.4$ and $0.8 \mathrm{~mm})$ were fabricated on an SOI platform, see Figure $4 \mathrm{e}$ and Supplementary Information. We measured the link power efficiency $\eta_{\mathrm{P}}=P_{\mathrm{RX}} / P_{\mathrm{TX}}\left(P_{\mathrm{RX}}\right.$ and $P_{\mathrm{TX}}$ are the received and transmitted power, respectively) as a function of $d$ by injecting light into standard 450-nm-wide strip waveguides (connected to the antennas) using conventional grating couplers and tapers (Figure 4a). The experimental antenna gain was also determined and found to be in good agreement with our theoretical estimation (Figure $4 \mathrm{~b}$ ). Digital data transmission over the $0.4-\mathrm{mm}$ link was demonstrated by sending $40 \mathrm{Gbit} \mathrm{s}^{-1}$ pseudo-random bit sequences at 38 different ITU channels evenly spaced by $0.8 \mathrm{~nm}$, ranging from 1534.25 to $1563.86 \mathrm{~nm}$ (a complete description of the employed set-up can be found in Supplementary Information). Clean eye diagrams (Supplementary Information) and bit error rates (BER, number of detected bit errors over the total number of transmitted bits) below $10^{-9}$, that is, sufficiently low to rule out forward-error correction schemes often related to an increase in chip complexity, were measured in all cases, verifying the abovementioned broadband features. This performance shows the feasibility of attaining terabitper-second data streaming over $\mathrm{mm}$-scale distances by using wavelength multiplexing. To illustrate this possibility, we transmitted $160 \mathrm{Gbit} \mathrm{s}^{-1}$ data streams consisting of four de-correlated 40-Gbit s ${ }^{-1}$ sequences at wavelengths ranging from $1548.52 \mathrm{~nm}$ to $1550.92 \mathrm{~nm}$ ( $0.8 \mathrm{~nm}$ evenly distributed), obtaining the same performance in terms of BER (Figure 4c). Remarkably, the demonstrated directive behavior could permit the implementation of unguided crossings in many different configurations (as in Figure 1) without perturbing the beam propagation or adding any undesired crosstalk (and even leads us to envisage the possibility of achieving efficient inter-core on-chip wireless interconnects), thus providing a much more flexible optical interconnect architecture.

Along this line, so far, research efforts have focused on optimizing single (two-waveguide) crossings, achieving an excellent performance 

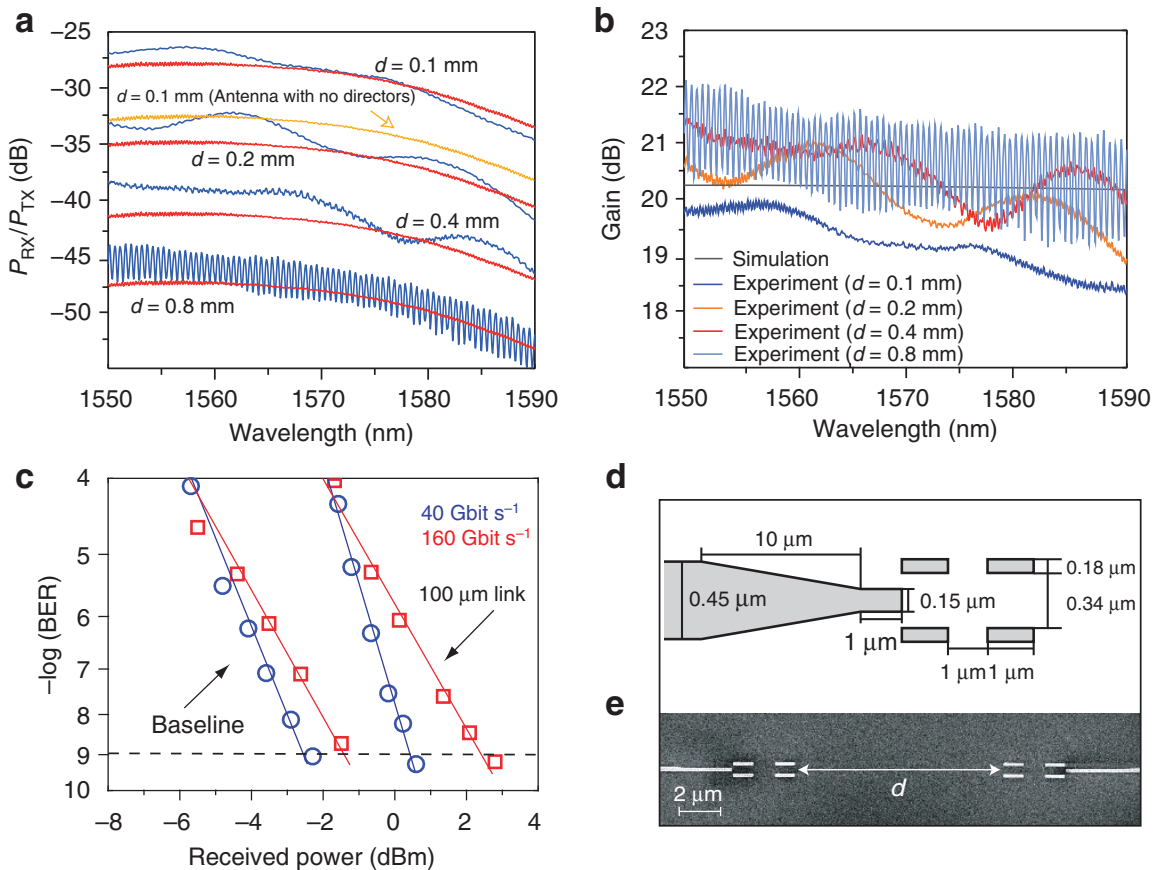

d

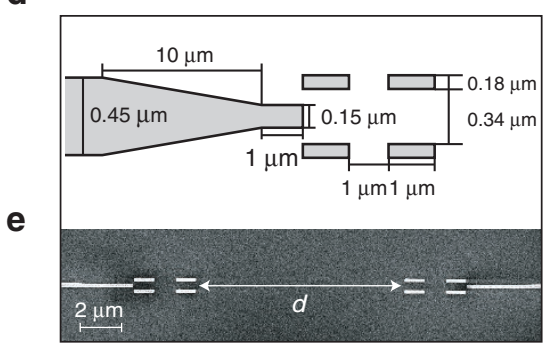

Figure 4 Experimental high-speed long-reach wireless data transmission. (a) Experimental (blue) and theoretical (red) power efficiency for different distances d. The theoretical efficiency is obtained as $\eta_{P}=\left(D \eta_{T} \eta_{G}\right)^{2} C_{R}$, where $D$ is the antenna directivity, $\eta_{G}$ accounts for the experimental grating coupler insertion losses, and $C_{R}$ for the reflections at the top and bottom $\mathrm{SiO}_{2}$ interfaces (Supplementary Information). For comparison purposes, the simulated efficiency corresponding to a bare tapered antenna with no directors is also included for the 100- $\mu \mathrm{m}$ link. (b) Simulated and experimental (retrieved from the measurements with the previous formula) antenna gain, defined as $G=D \eta_{T}$. (c) BER measured with an error analyzer and evaluated as a function of the decreasing optical power received by the photodetector for a baseline configuration where the wireless link was replaced with a silicon straight waveguide (left) and for a 100- $\mu \mathrm{m}$ wireless link (right). The $160 \mathrm{Gbit} \mathrm{s}^{-1}$ BER corresponds to the demodulation of channel 34 when transmitting channels 33 to 36. The crosstalk produced by surrounding channels $(33,35$ and 36) introduces low penalties. (d) Schematic top view of the antenna and directors. (e) SEM image of a point-to-point link with $d=10 \mu \mathrm{m}$.

both in terms of crosstalk and insertion loss ${ }^{34}$. Although an equivalent wireless crossing could show a negligible crosstalk (due to the highdirectivity of the proposed antennas), it would be extremely difficult to surpass the insertion loss performance obtained with state-of-the-art guided approaches. The potential of the wireless approach, however, becomes apparent in more complex configurations involving a high number of ports. In this case, the solution adopted in guided technology consists of a combination of many single crossings ${ }^{5,34}$. Unless using rectangular grids (crossing of two arrays of parallel waveguides), this results in complex topologies with different drawbacks. Consider, for instance, the configuration in Figure 5a. It consists of six input waveguides whose outputs are vertically flipped. This configuration can also be understood as three single crossings (red, green and blue) that at the same time, cross each other, see Figure 5b. Even with a crossing-aware routing algorithm, a guided implementation of this device would require a large number of crossings and bends ${ }^{5}$, yielding either a large footprint or high values of crosstalk and insertion loss. To reduce the footprint, the best option is to use a direct star crossing, whether wireless as in Figure $5 \mathrm{c}$ or guided as in Figure 5d. To analyze these alternatives, we fabricated and optically characterized both (same antenna configuration as in the data transmission example). It is worth mentioning that we have not found any previous work on multiple simultaneous crossings with more than four ports. Hence, we have used a direct crossing for the guided device. In any case, due to the small (and fixed, if a symmetric behavior is desired) angle between adjacent waveguides, it would be challenging to optimize this crossing using tapered waveguides as in the single crossing case. The measured device transmission efficiency between the input port and the output ports located at $0^{\circ}$ (direct interconnect), $30^{\circ}, 60^{\circ}$ and $90^{\circ}$ is shown in Figure $5 \mathrm{c}$ and $5 \mathrm{~d}$. Note that the wireless signal transmitted to the output ports at $120^{\circ}$ and $150^{\circ}$ was so low that it was outside the detector range. Regarding the crosstalk, it is evident that the antenna-based configuration outperforms its guided counterpart. Specifically, looking at the most critical output ports (those at $\pm 30^{\circ}$ ), a crosstalk figure between -10 and $-24 \mathrm{~dB}$ (mean value of $-13 \mathrm{~dB}$ ) is obtained within the experimental window for the wireless crossing, while unacceptable crosstalk values between 0 and $-6 \mathrm{~dB}$ (mean value of $-2.9 \mathrm{~dB}$ ) are obtained for the guided case. Moreover, the direct interconnect transmission efficiency (or, equivalently, the insertion loss) is similar in both cases, which is explained by the much higher crosstalk and scattering of the guided device. Therefore, the better performance and compact feature (facing antennas separated by $10 \mu \mathrm{m}$ ) of the wireless solution provides a superior solution for footprint-constrained applications.

The increasingly indispensable requirement for reconfigurable onchip networks motivated a second application: a dynamically tunable antenna beam-steering system. To this end, we benefited from a distinctive feature of the proposed architecture, that is, the excitation scheme based on silicon waveguides. The fact that the refractive index $n$ of these structures can be modified dynamically with different effects (for example, thermo-optic, electro-optic) permits the independent tuning of the phase (and ultimately the amplitude via interferometry) of each waveguide's output signal and, therefore, the phase of the antenna it feeds. Consequently, the use of antenna arrays ${ }^{32}$ in combination with any of these effects makes it possible to mold the in-plane radiation diagrams in almost any desired way. In particular, 
a

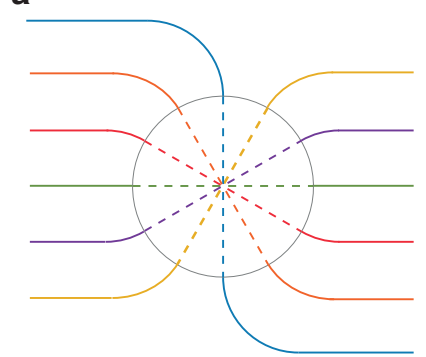

b

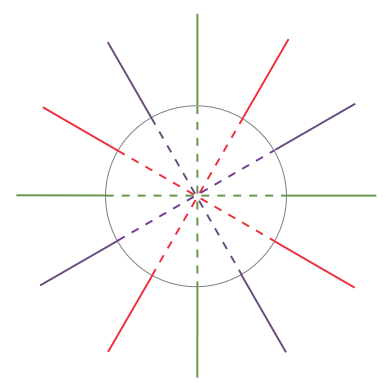

C
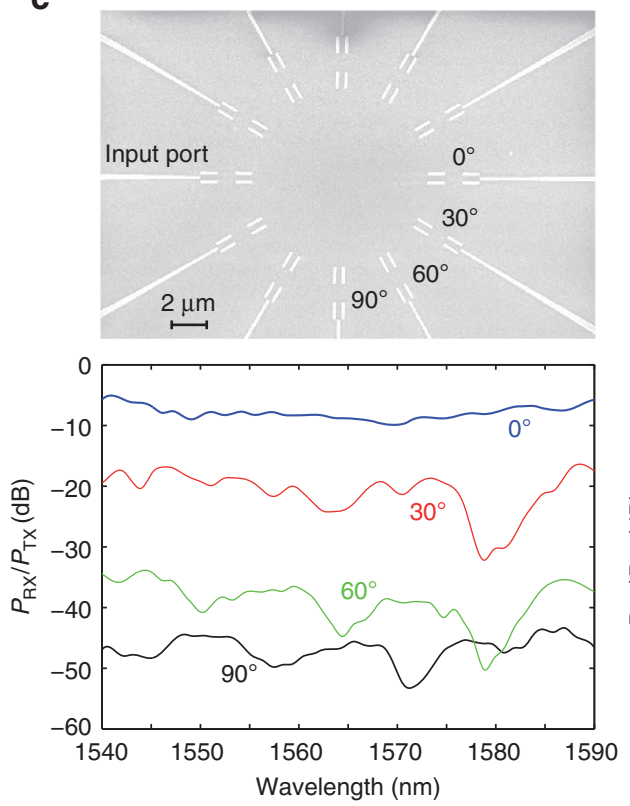

d
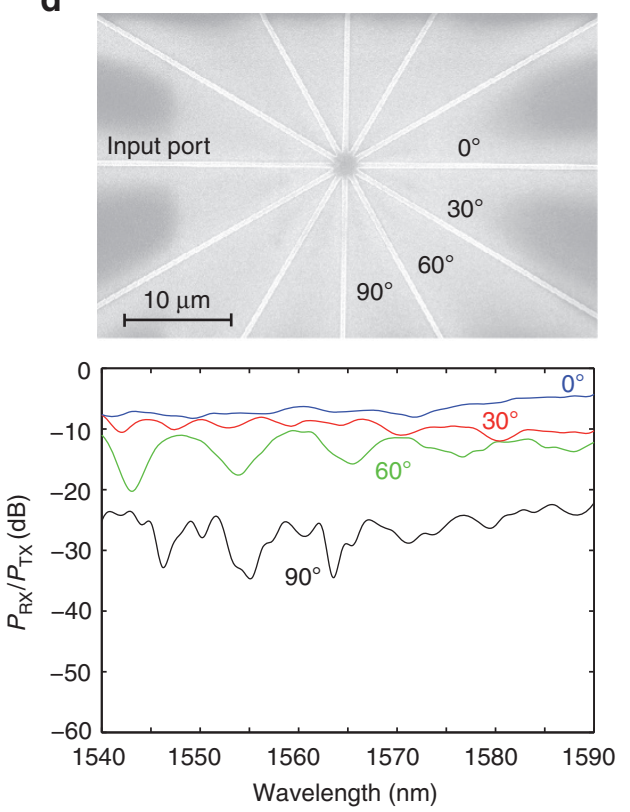

Figure 5 Comparison of wireless and guided 12-port crossings. (a, b) Two different utilities of the device. (c, d) SEM images and measured transmission efficiency for the receiving ports at $0^{\circ}, 30^{\circ}, 60^{\circ}$ and $90^{\circ}$ associated with the fabricated (c) wireless and (d) guided implementations of the device (that is, dashed connections inside the gray circles).

an array in which all elements are fed with the same amplitude and an $\alpha$ progressive phase delay shows a maximum in the direction $\theta_{\max }$, with $\alpha=-k d_{\mathrm{A}} \cos \theta_{\max }\left(d_{\mathrm{A}}\right.$ representing the separation between neighboring antennas). Thus, we can steer the radiated beam at any direction through a proper choice of $\alpha$. As an example, we exploited silicon's thermo-optic effect ${ }^{35}$ to achieve an active control of a fourelement array (separation between emitting antennas $d_{\mathrm{A}}=1.65 \mu \mathrm{m}$ ). Note that as the single-antenna directivity increases, the maximum directivity of the full array decreases more quickly with $\left|\theta_{\max }\right|$, limiting the device angular range. However, low-directivity antennas result in low full-array directivities even for small values of $\theta_{\max }$, increasing the crosstalk between adjacent receiving antennas. Finally, we used bare inverted-taper antennas, as this configuration yields very high directivities (Supplementary Information) and enables us to steer the beam by $\pm 15^{\circ}$ with a maximum intensity radiation drop of $\sim 3 \mathrm{~dB}$. This $30^{\circ}$ range, together with the high link distances enabled by such high directivities allows us to place a considerable number of receiving antennas at different angles. As reception always occurs at normal incidence, we employed an inverted taper +2 director configuration for the receiving antennas (Figure $6 \mathrm{~b}$ and $6 \mathrm{c}$ ). After covering the fabricated antennas and waveguides with a $1-\mu$ m-thick $\mathrm{SiO}_{2}$ layer, we deposited a 100-nm-thick titanium nanowire or nanoheater (terminated by two pads) on top of each feeding waveguide (Figure 6a). The electrical current flowing through each nanoheater is converted to heat via the Joule effect, which propagates through the $\mathrm{SiO}_{2}$ layer down to the corresponding silicon waveguide, modifying its local temperature $(T)$, and inducing a refractive index change given by $\partial n / \partial T=1.86 \times 10^{-4} \mathrm{~K}^{-1}$. The temperature variation is determined by the intensity of the electrical current, which is controlled by the voltage applied across the corresponding pads. With this technique, we experimentally demonstrated a reconfigurable $30^{\circ}$ beam-steering system over $100-\mu \mathrm{m}$ links $(\lambda=1550 \mathrm{~nm})$. This steering device can be regarded as a 1-to-3-port switch. Along this line, we estimated its switching time: $5.2 \mu \mathrm{s}$, and its switching power: $8.3 \mathrm{~mW}$ per nanoheater (three simultaneous active heaters are required to aim the beam at any of the side antennas). This results in a figure of merit (switching power times the switching time) of $43.16 \mathrm{~mW} \cdot \mu \mathrm{s}$, which is in agreement with current state of the art (Supplementary Information). The aim of this demonstration is to illustrate the capabilities of the proposed reconfigurable wireless links in terms of reach and angular range and therefore the device was not optimized to work as a switch. In this sense, there are several aspects that can be easily improved by a proper choice of the system parameters. First, the $3 \mathrm{~dB}$ relative power loss between adjacent ports can be reduced by decreasing the steering angular range (for example, by increasing $d_{\mathrm{A}}$ ). Second, although the sharp beams radiated by our highly directive antennas yield a crosstalk value below $-8 \mathrm{~dB}$ (Figure 6d), which would be acceptable for some applications (for example, by using forward-error correction schemes), it would not be for others. Here, we can achieve a dramatic improvement by exploiting an important feature of antenna arrays. In particular, the directivity of an array of $N$ antennas is periodic as a function of $\psi=k d_{\mathrm{A}} \cos \theta$, with $N-1$ equally spaced zeros per period. The phase delay $\alpha$ shifts the directivity diagram in $\psi^{32}$. Hence, there are $N$ values of $\alpha$ associated with $N$ different principal radiation directions, for each of which the radiated power is nearly maximum, while being zero for the remaining $N-1$ directions. Therefore, by using $N$-antenna arrays, we can theoretically obtain a $1 \times N$ switch with no crosstalk. Lastly, the link distance and the separation between receiving antennas can be considerably reduced to minimize the footprint and the radiation loss.

To illustrate these ideas, we designed and numerically simulated two different optimized switches. In both cases, we considered a value of $d_{\mathrm{A}}=2.2 \mu \mathrm{m}$ and a spacing of $3 \mu \mathrm{m}$ between adjacent receiving antennas. As a first example, we demonstrate that the proposed 4 -antenna array can be easily tuned to build a high-performance $1 \times 4$ switch by placing the receiving antennas at the angles shown in Figure 6e, which depicts the total array gain for each optimum switch state (value of $\alpha$ ). As can be observed, a negligible crosstalk with less than $1 \mathrm{~dB}$ relative loss is obtained. It is clear that fabrication imperfections will result in a non-zero crosstalk. However, the good 
a

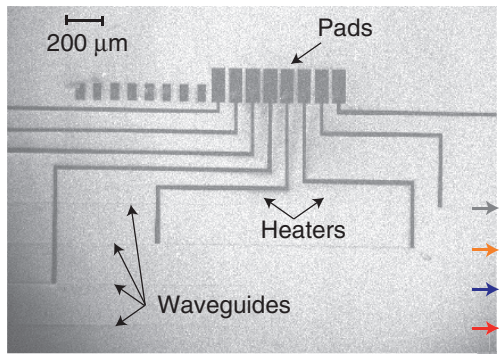

d

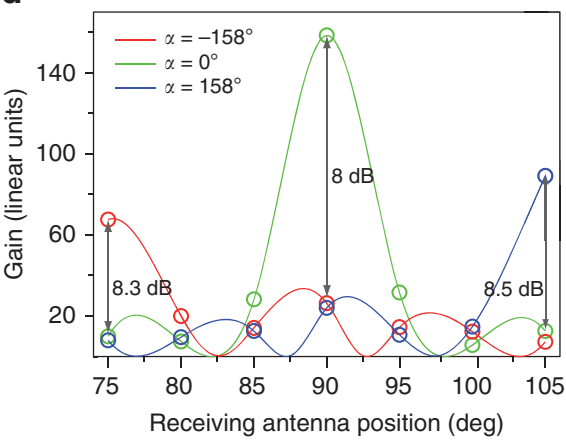

e 30

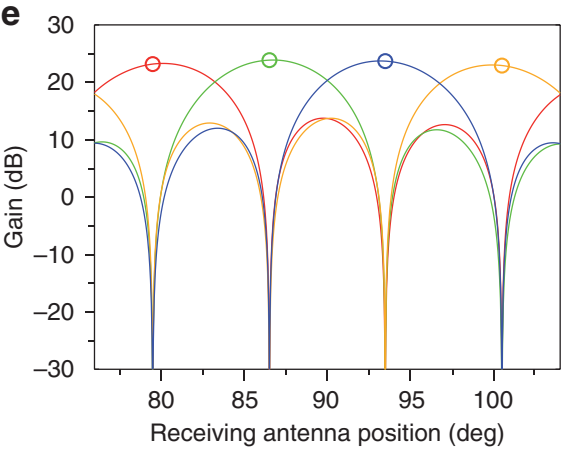

b

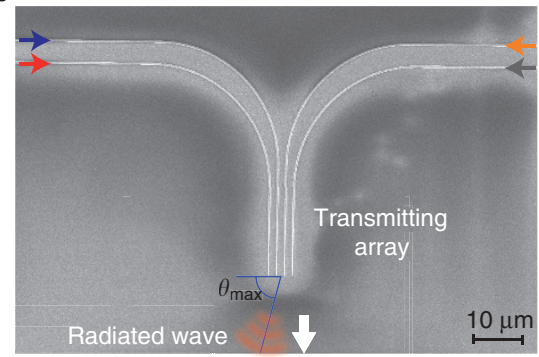

C

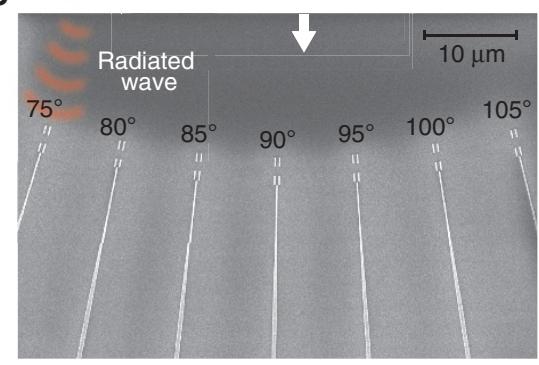

f

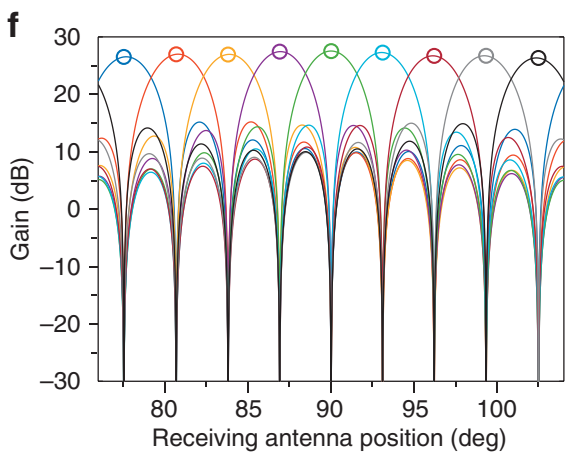

Figure 6 Experimental electrically reconfigurable beam steering. (a) SEM image of the nanoheaters with their corresponding pads and the underlying feeding waveguides. (b) SEM image of the waveguides used to feed the 4-antenna emitting array (colored arrows indicate the waveguides paths in a and b). (c) SEM image of the receiving subsystem consisting of seven antennas placed at different angles. The antennas at $75^{\circ}, 90^{\circ}$ and $105^{\circ}$ are taken as the device output ports. The values of $\alpha$ that allow us to steer the beam to each of these ports were estimated to be $-158^{\circ}, 0^{\circ}$ and $158^{\circ}$, respectively (Supplementary Information). (d) Total array gain associated with each receiving antenna $(d=100 \mu \mathrm{m}, \lambda=1550 \mathrm{~nm})$ for the three values of $\alpha$. Circles indicate measured values at each antenna position. The crosstalk at the output ports is highlighted, with values always higher than $8 \mathrm{~dB}$. (e, f) Simulated total array gain associated with two different wireless $1 \times N$ switches for each of the $N$ optimized values of $\alpha$ (each with a different color). The zeros of the corresponding radiation diagrams determine the $N$ angles at which the receiving antennas are located. At each of these positions, the radiated power is zero for all values of $\alpha$ except one, for which the radiated power is almost maximum (marked with a circle). (e) $N=4$. (f) $N=9$.

agreement between our theoretical calculations and the experimental results obtained for the fabricated switch confirms that our simulations constitute a good prediction of the device's experimental performance. Hence, very low crosstalk is expected for this new design. The employed receiving antenna separation and angle span determine a link distance of $d=23 \mu \mathrm{m}$, yielding a wireless subsystem footprint (including the antennas) of $\sim 48 \times 14 \mu \mathrm{m}^{2}$. As a second example, we designed a $1 \times 9$ switch with similar features and a corresponding footprint of $80 \times 30 \mu \mathrm{m}^{2}(d=55 \mu \mathrm{m})$, see Figure $6 f$.

To calculate the overall footprint, the heated regions must also be taken into account. However, it should be noted that our work is focused on the photonic subsystem and we did not optimize the heating subsystem. Nonetheless, our device has no special heating requirements, and any state-of-the-art heater designed for a typical strip waveguide is also valid for our system. For instance, considering the heaters of Ref. 36, a rough estimation provides a total footprint of $45 \times 113 \mu \mathrm{m}^{2}$ (this value is approximately three times lower than the most compact design we have found in the literature ${ }^{36-38}$ ) and $120 \times 155 \mu^{2}$ for the $1 \times 4$ and $1 \times 9$ switches, respectively.

There are two additional potential advantages of our approach. First, our system only requires one stage regardless of the number of output ports. The guided approach is based on the use of cascaded $1 \times 2$ switches $^{36}$, for which crosstalk becomes preponderant and cumulative, swiftly degrading the system performance only after a few stages $^{39}$. Consequently, the wireless switch is more versatile and easily scalable. For example, to increase by one output, a $1 \times 8$ switch requires passing from 3 to 4 stages, whereas in the wireless case, only adding one antenna to the input and output arrays is required. Furthermore, unless the number of outputs is a power of 2, the signal will go through a different number of stages depending on the output 

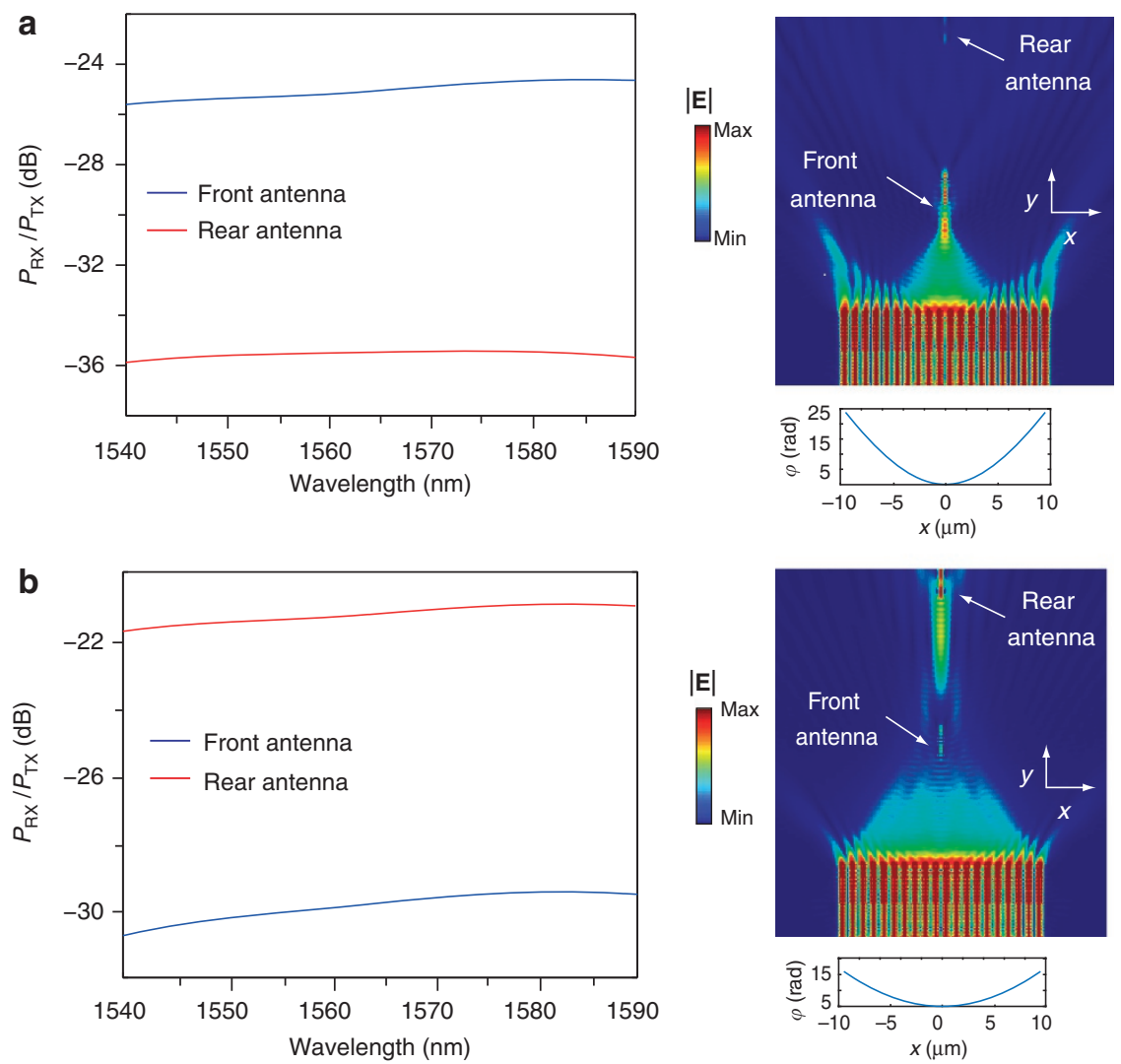

Figure 7 Depth-selective bridging transmission scheme (CST simulations) (a, b). By varying the input phases $\varphi$ of a 20-antenna array, radiated energy can be focused not only at different angles (as in beam steering) but also at different depths. As an example, two receiving antennas are placed at (a) $(x=0, y=10)$ $\mu \mathrm{m}$ and $(\mathbf{b})(x=0, y=25) \mu \mathrm{m}$. Depending on the employed phase profile, the radiated power is sent to the front or rear antenna, see left column. The $E$-field distribution $(\lambda=1550 \mathrm{~nm})$ and the associated phase profile for both configurations is shown in the right column.

port in a guided switch, which will give rise to an additional unbalance. Second, in the proposed approach, the heating and wireless subsystems are separated. This can simplify the packaging of the device in comparison to the guided solution, in which the heaters are distributed throughout the whole system.

It has been shown that the radiative crosstalk between neighboring elements may be important in guided devices because of structure imperfections ${ }^{40,41}$. Therefore, a discussion regarding the radiative crosstalk associated with wireless components naturally follows. There are two main factors suggesting that this parameter could be negligible in most situations. First, the devices outside a given antenna angular range are not particularly affected as the radiated power rapidly decreases away from the principal radiation direction (for example, the gain of a two-director antenna falls by almost $20 \mathrm{~dB}$ at $\sim 20^{\circ}$ ). Second, for the devices within this range, it must be considered that the radiated power decreases as $1 / d^{2}$. At $d=20 \mu \mathrm{m}$, the power efficiency at $0^{\circ}$ for a single two-director antenna is below $-20 \mathrm{~dB}$. In our wireless devices, this is compensated by using a high-directivity receiving antenna ( $>20 \mathrm{~dB}$ ). However, typical guided devices will exhibit a very low directivity and collect almost no radiated energy.

To further illustrate the possibility of controlling radiated beams via wireless silicon photonics, we theoretically developed a device able to generate reconfigurable tailor-made interference patterns. To this end, we designed a 20-element array of a new configuration of tapered antennas with a tip width of $300 \mathrm{~nm}$, engineered to exhibit a directivity of $\sim 10 \mathrm{~dB}$. This lower directivity value is more convenient in this case to favor the creation of multiple-interference waves and fully exploit the optical antenna array concept. We concentrated on achieving light focusing with a reconfigurable focal point position by imposing a hyperbolic phase profile on the emitting array antennas (Supplementary Information). Such a device can confine radiated energy not only in angle but also in depth, as shown in the example of Figure 7. Here, we show that by tuning the phase of the antennas, it is possible to send radiated energy either to a nearby detector or to a second one behind it (in which case the first detector is bridged), with a crosstalk of $\sim-10 \mathrm{~dB}$ in both cases. Consequently, one could implement functionalities not addressable by guided photonics, such as on-chip 2D-selective optical detection schemes (as opposed to the $1 \mathrm{D}$-selective capacities of beam-steering devices), radiating behind 'obstacles', or 2D-space mapping.

As a final example, we conducted an additional experiment to demonstrate the potential of the proposed wireless architecture for lab-on-a-chip applications. Inspired by the technique employed in flow cytometry ${ }^{7}$, we designed and fabricated an on-chip hydrodynamic parametric analysis system in which different types of microparticles can flow through a microfluidic channel and be simultaneously illuminated by a narrow beam. The main goal was to classify the flowing particles according to their unique time-dependent scatteredfield signatures. To this end, we incorporated an optical subsystem into the chip consisting of a highly directive emitting antenna (inverted taper +2 directors) and two identical receiving antennas connected to standard strip waveguides (Figure 8a). One of the receiving antennas collects the power at $90^{\circ}$ and is used for alignment/ calibration purposes. The second receiving antenna measures the 
a

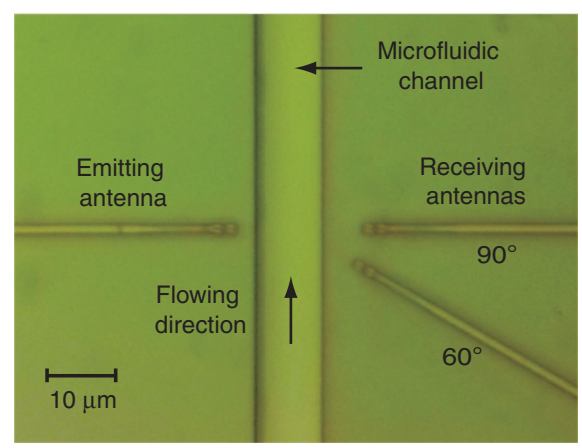

b

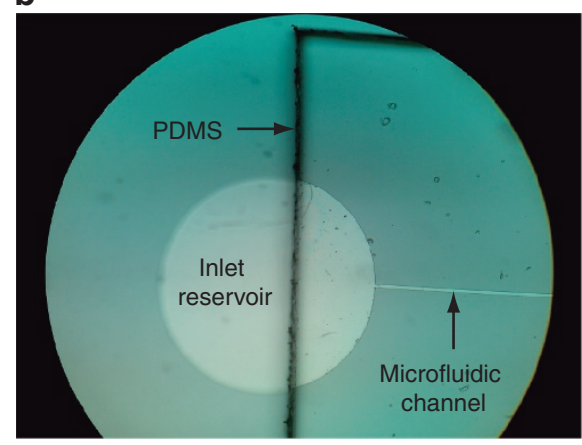

C

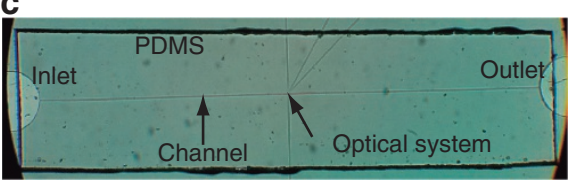

d
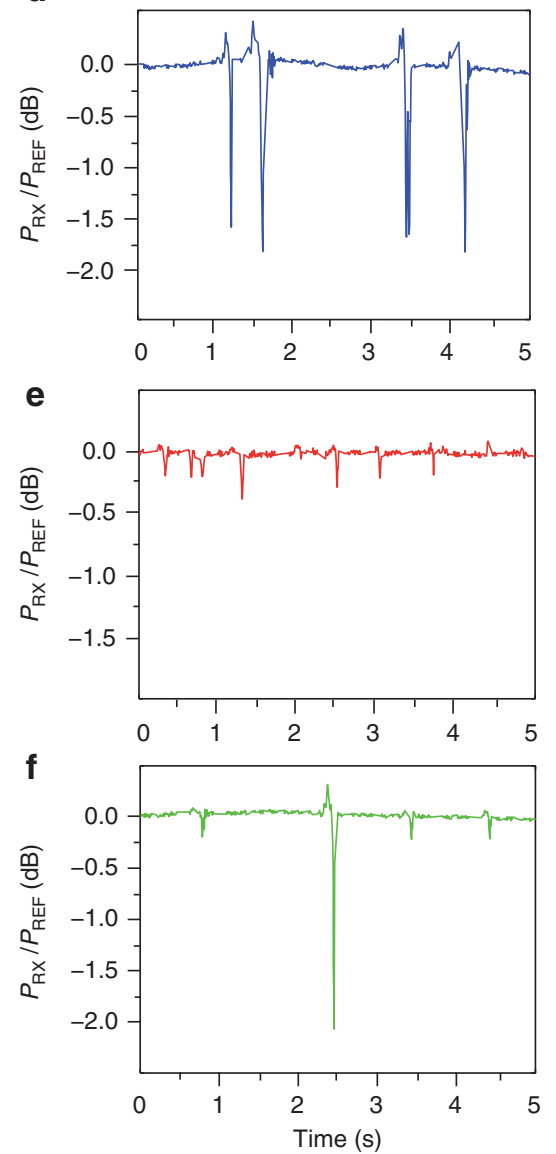

Figure 8 Experimental flow cytometer for microparticle characterization. (a) Optical microscope image of the fabricated device. (b) Optical microscope image of the inlet reservoir and part of the microfluidic channel. The border of the top PDMS layer can also be observed. (c) Complete microfluidic system. The position of the optical system (not visible at this scale) is also indicated. (d-f) Power time-dependent efficiency measured at $60^{\circ}$ for $2-\mu \mathrm{m}(\mathrm{d}), 1-\mu \mathrm{m}(\mathrm{e})$, and a mixed solution of polystyrene microspheres (f). The received power when no particles are flowing is taken as the reference $\left(P_{\mathrm{REF}}\right)$.

scattered field at $60^{\circ}$, which enables us to classify the particles. In addition, a microfluidic channel was built to allow the particles to flow in between the antennas. To build the channel, a $10-\mu \mathrm{m}$-wide $4-\mu \mathrm{m}$ deep trench was opened in the $\mathrm{SiO}_{2}$ region between the emitting and receiving antennas, and subsequently sealed by placing a $150-\mu \mathrm{m}$ polydimethylsiloxane layer atop (Figure $8 \mathrm{~b}$ ). A couple of inlet and outlet reservoirs providing access to the channel completes the microfluidic subsystem (Figure 8c). To test the device, we used polystyrene microspheres (a standard benchmark for calibrating flow cytometers) with two different diameters ( 1 and $2 \mu \mathrm{m})$ in an aqueous solution with a concentration of 1:100 (2\% solids). Capillary forces ensure that the solution fills the channel and the microspheres flow between the antennas when casting a solution drop into the inlet reservoir. To learn the characteristic scattered-field signature of each kind of microsphere, we prepared two different solutions including either 1- $\mu \mathrm{m}$ spheres or $2-\mu \mathrm{m}$ spheres alone. Both signatures appear in the form of a dip, of $\sim 2 \mathrm{~dB}$ in the case of the large microspheres and $0.25 \mathrm{~dB}$ in the case of the small ones (Figure $8 \mathrm{~d}$ and $8 \mathrm{e}$ ). The signatures are exclusive, as no $2(0.25) \mathrm{dB}$ peaks were observed when flowing the $1-\mu \mathrm{m}(2-\mu \mathrm{m})$ sphere. Finally, a flow of an equally mixed solution containing both types of particles was used (Figure 8). In this case, we were able not only to detect when a particle goes through the optical link (due to the presence of a dip) but also easily classify the particle, that is, identify its size, depending on the magnitude of the measured dip. As the optical system is not in physical contact with the target, it can be reused an unlimited number of times.

Currently, there are two main approaches to microflow cytometers. The first one uses optical fibers to illuminate the particles and collect the scattered field ${ }^{8,42}$, resulting in bulky devices with potential losses and failure of discrete component interfaces. A second more compact and integrated approach uses focusing components, such as lenses or multimode interference devices (to achieve a reduced beam waist and resolve small particles) with a typical lateral size in the mm-scale ${ }^{42-44}$. Outstandingly, the directive nature of our illuminating antenna implies that low-divergent focused beams can be generated (the beam full width at half maximum for our experimental configuration is $\sim 4 \mu \mathrm{m}$ at the center of the channel). This allows us to resolve very small particles $(1-\mu \mathrm{m}$ diameter microspheres remain as the smallest target detected so far with integrated on-chip devices) without requiring large focusing structures ${ }^{42}$, which entails a promising solution for sub-micron particle detection. Therefore, the proposed device constitutes a low-cost chip-integrated type of flow cytometer, whose main advantage over existing solutions is its extremely reduced footprint (only a few $0.64 \times 14 \mu \mathrm{m}^{2}$ antennas), while exhibiting stateof-the-art precision. Specifically, a dramatic footprint reduction of four orders of magnitude with respect to the most compact previous designs is achieved ${ }^{42-44}$, significantly minimizing fabrication time, energy and potential errors. Moreover, the absence of optical fibers in 
our device simplifies both the packaging and the automation of the manufacturing process. The use of SOI fully integrated structures enables the possibility of easy fabrication, assuring a prompt mass production of point-of-care devices. Finally, the high density of detectors allowed by the small size of the antennas permits a finer scattered-field sampling angular resolution, resulting in a more accurate classification system.

A final comment on the role of the employed antenna directors: Although the measured $\sim 5$-dB link-efficiency enhancement provided by these elements $(\sim 3 \mathrm{~dB}$ per antenna according to our calculations, see Figure 3b) could have a limited impact on the global system performance for long links with high propagation losses (for example, with $\eta_{\mathrm{P}} \approx 47 \mathrm{~dB}$ for $d=0.8 \mathrm{~mm}$ ), for short links with low propagation losses (for example, for crossings or short-link switches), a 5-dB improvement can be crucial, for example, to fulfill a given BER specification. In addition, the enhancement provided by the directors affects other important features of the proposed devices. For instance, a bare inverted-taper antenna exhibits a broader radiation pattern and, consequently, introduces higher crosstalk values in applications such as the proposed crossing structure (the crosstalk at $30^{\circ}$ associated with the inverted-taper antenna without directors would be $\sim 6 \mathrm{~dB}$ higher than that associated with the 2-director antenna). For the same reason, the directors also influence the lateral size of the radiated beam. For example, without the directors, the beam waist in the studied cytometry application would increase from 4 to $6 \mu \mathrm{m}$, considerably limiting the minimum resolvable particle size.

\section{CONCLUSIONS}

The proposed model of a wireless optical system provides a CMOScompatible on-chip platform supporting a wide range of application scenarios that are less manageable using traditional guided architectures or previous plasmonic or dielectric antenna configurations. In addition to the construction of flexible and reconfigurable networks for classical and quantum HPCs and high-speed communications, these systems could find applications in other fields, such as the generation of complex beams for material processing and optical tweezing ${ }^{10}$. From a lab-on-a-chip perspective, the compact and versatile nature of the proposed approach could facilitate a high density, robust and resilient integration of different laboratory equipment - in addition to flow cytometry, dynamic light scattering, Raman spectroscopy and gas chromatography are interesting possibilitiestogether with signal processing systems and other functionalities on the same chip (with the potential of performing a large number of simultaneous measurements), that paves the way to point-of-care clinical diagnosis and portable chemical, biological or medical research equipment.

\section{CONFLICT OF INTEREST}

The authors declare no conflict of interest.

\section{AUTHOR CONTRIBUTIONS}

CG-M and SL proposed the new dielectric antenna concept, developed the theory, designed and conducted the experiments, analyzed the data and prepared the manuscript. $\mathrm{AB}$ designed the experimental modulation set-up. AG and LB developed the fabrication process of the silicon samples. AB, SM and LS contributed to the simulations and experiments. NSL fabricated the PDMS layers and participated in the development of the sensing experiment. JM conceived and proposed the concept of multipurpose on-chip wireless optical systems, analyzed the data and suggested improvements to the manuscript. All authors discussed the results.

\section{ACKNOWLEDGEMENTS}

Funding from grant TEC2015-63838-C3-1-R OPTONANOSENS (MINECO/ FEDER, UE) is acknowledged. This work was also supported by project TEC2015-73581-JIN (AEI/FEDER, UE), the EU-funded projects FP7-ICT PHOXTROT (No.318240) and H2020-, the EU-funded H2020-FET-HPC EXANEST (No.671553) and the Generalitat Valenciana's PROMETEO grant NANOMET PLUS (PROMETEO II/2014/34) CG-M acknowledges support from Generalitat Valenciana's VALi+d postdoctoral program (exp. APOSTD/ 2014/044). We thank David Zurita for his help in the design of the data acquisition code for the sensing application.

1 Kirchain R, Kimerling R. A roadmap for nanophotonics. Nat Photonics 2007; 1: 303-305.

2 Fan XD, White IM. Optofluidic microsystems for chemical and biological analysis. Nat Photonics 2011; 5: 591-597.

3 Zhuang LM, Roeloffzen CGH, Meijerink A, Burla M, Marpaung DAl et al. Novel ring resonator-based integrated photonic beamformer for broadband phased array receive antennas-part II: experimental prototype. J Lightw Technol 2010; 28: 19-31.

4 Yu NF, Capasso F. Flat optics with designer metasurfaces. Nat Mater 2014; 13: 139-150.

5 Condrat C, Kalla P, Blair S. Crossing-aware channel routing for integrated optics. IEEE Trans Comput-Aided Design Integr Circuits Syst 2014; 33: 814-825.

6 Lee BG, Rylyakov AV, Green WMJ, Assefa S, Baks CW et al. Monolithic silicon integration of scaled photonic switch fabrics, CMOS logic, and device driver circuits. J Lightw Technol 2014; 32: 743-751.

7 Robinson JP, Roederer M. Flow cytometry strikes gold. Science 2015; 350: 739-740.

8 Mao XL, Nawaz AA, Lin SC, Lapsley MI, Zhao YH et al. An integrated, multiparametric flow cytometry chip using 'microfluidic drifting' based three-dimensional hydrodynamic focusing. Biomicrofluidics 2012; 6: 024113.

9 Schurr JM. Dynamic light scattering of biopolymers and biocolloids. CRC Crit Rev Biochem 1977; 4: 371-431.

10 Padgett M, Bowman R. Tweezers with a twist. Nat Photonics 2011; 5: 343-348.

11 Haurylau M, Chen GQ, Chen H, Zhang JD, Nelson NA et al. On-chip optical interconnect roadmap: challenges and critical directions. IEEE J Select Top Quantum Electron 2006; 12: 1699-1705.

12 Chan JN, Hendry G, Biberman A, Bergman K. Architectural exploration of chip-scale photonic interconnection network designs using physical-layer analysis. J Lightw Technol 2010; 28: 1305-1315.

13 Vlasov Y, Green WMJ, Xia FN. High-throughput silicon nanophotonic wavelengthinsensitive switch for on-chip optical networks. Nat Photonics 2008; 2: 242-246.

14 Novotny L, van Hulst N. Antennas for light. Nat Photonics 2011; 5: 83-90.

15 Fischer H, Martin OJF. Engineering the optical response of plasmonic nanoantennas. Opt Express 2008; 16: 9144-9154.

16 Dregely D, Taubert R, Dorfmüller J, Vogelgesang R, Kern K et al. 3D optical Yagi-Uda nanoantenna array. Nat Commun 2011; 2: 267.

17 Ni XJ, Emani NK, Kildishev AV, Boltasseva A, Shalaev VM. Broadband light bending with plasmonic nanoantennas. Science 2012; 335: 427.

18 Koenderink AF, Alù A, Polman A. Nanophotonics: shrinking light-based technology. Science 2015; 348: 516-521.

19 Polman A. Plasmonics applied. Science 2008; 322: 868-869.

20 Brongersma ML, Shalaev VM. The case for plasmonics. Science 2010; 328: 440-441.

21 Alù $A$, Engheta $N$. Wireless at the nanoscale: optical interconnects using matched nanoantennas. Phys Rev Lett 2010; 104: 213902.

22 Solís DM, Taboada JM, Obelleiro F, Landesa L. Optimization of an optical wireless nanolink using directive nanoantennas. Opt Express 2013; 21: 2369-2377.

23 Dregely D, Lindfors K, Lippitz M, Engheta N, Totzeck M et al. Imaging and steering an optical wireless nanoantenna link. Nat Commun 2014; 5: 4354.

24 Curto AG, Volpe G, Taminiau TH, Kreuzer MP, Quidant R et al. Unidirectional emission of a quantum dot coupled to a nanoantenna. Science 2010; 329: 930-933.

25 Sun J, Timurdogan E, Yaacobi A, Hosseini ES, Watts MR. Large-scale nanophotonic phased array. Nature 2013; 493: 195-199.

26 Van Acoleyen K, Bogarets W, Jágerská J, Le Thomas N, Houdré R et al. Off-chip beam steering with a one-dimensional optical phased array on silicon-on-insulator. Opt Lett 2009; 34: 1477-1479.

27 Van Acoleyen K, Rogier H, Baets R. Two-dimensional optical phased array antenna on silicon-on-insulator. Opt Express 2010; 23: 13655-13660.

28 Rodríguez-Fortuño FJ, Puerto D, Griol A, Bellieres L, Martí J et al. Sorting linearly polarized photons with a single scatterer. Opt Lett 2014; 39: 1394-1397.

29 Krasnok AE, Miroshnichenko AE, Belov PA, Kivshar YS. All-dielectric optical nanoantennas. Opt Express 2012; 20: 20599-20604.

30 Filonov DS, Krasnok AE, Slobozhanyuk AP, Kapitanova PV, Nenasheva EA et al. Experimental verification of the concept of all-dielectric nanoantennas. Appl Phys Lett 2012; 100: 201113.

31 Cárdenas J, Poitras $\mathrm{CB}$, Robinson JT, Preston $\mathrm{K}$, Chen $\mathrm{L}$ et al. Low loss etchless silicon photonic waveguides. Opt Express 2009; 17: 4752-4757.

32 Balanis CA. Antenna Theory: Analysis and Design. Wiley: New York, 1982. 
33 Kosako T, Kadoya Y, Hofmann HF. Directional control of light by a nano-optical Yagi-Uda antenna. Nat Photonics 2010; 4: 312-315.

34 Subbaraman $\mathrm{H}$, Xu XC, Hosseini A, Zhang XY, Zhang Y et al. Recent advances in siliconbased passive and active optical interconnects. Opt Express 2015; 23: 2487-2511.

35 Della Corte FG, Esposito Montefusco M, Moretti L, Rendina I, Cocorullo G. Temperature dependence analysis of the thermo-optic effect in silicon by single and double oscillator models. J Appl Phys 2000; 88: 7115-7119.

36 Chu T, Yamada H, Ishida S, Arakawa Y. Compact $1 \times N$ thermo-optic switches based on silicon photonic wire waveguides. Opt Express 2005; 13: 10109-10114.

37 Wang WJ, Zhao Y, Zhou HF, Hao YL, Yang JY et al. CMOS-compatible $1 \times 3$ silicon electrooptic switch with low crosstalk. IEEE Photon Technol Lett 2011; 23: 751-753.

38 Cui KY, Zhao Q, Feng X, Liu F, Huang YD et al. Ultra-compact and broadband $1 \times 4$ thermo-optic switch based on W2 photonic crystal waveguides. Proceedings of 2005 Opto-Electronics and Communications Conference; 28 June-2 July 2015; Shanghai, IEEE: Shanghai 2015.

39 Lee BG, Dupuis N, Pepeljugoski P, Schares L, Budd R et al. Silicon photonic switch fabrics in computer communications systems. J Lightw Technol 2015; 33: 768-777.

40 Song WW, Gatdula R, Abbaslou S, Lu M, Stein A et al. High-density waveguide superlattices with low crosstalk. Nat Commun 2015; 6: 7027.
41 Melati D, Morichetti F, Gentili GG, Melloni A. Optical radiative crosstalk in integrated photonic waveguides. Opt Lett 2014; 39: 3982-3985

42 Zhang YS, Watts BR, Guo TY, Zhang ZY, Xu CQ et al. Optofluidic device based microflow cytometers for particle/cell detection: a review. Micromachines 2016; 7: 70.

43 Kotz KT, Petrofsky AC, Haghgooie R, Granier R, Toner M et al. Inertial focusing cytometer with integrated optics for particle characterization. Technology (Singap World Sci) 2013; 1: 27-36.

44 Hunt HC, Wilkinson JS. Multimode interference devices for focusing in microfluidic channels. Opt Lett 2011; 36: 3067-3069.

(c) (i) (2) (2) This work is licensed under a Creative Commons AttributionBY NC SA NonCommercial-ShareAlike 4.0 International License. The images or other third party material in this article are included in the article's Creative Commons license, unless indicated otherwise in the credit line; if the material is not included under the Creative Commons license, users will need to obtain permission from the license holder to reproduce the material. To view a copy of this license, visit http:// creativecommons.org/licenses/by-nc-sa/4.0/

(C) The Author(s) 2017

Supplementary Information for this article can be found on the Light: Science \& Applications' website (http://www.nature.com/lsa). 